

\title{
Comparison of Thermoluminescence Dosimeter's Response to Neutron and Gamma in a Mixed Radiation Field
}

\author{
Nabil M. Hassan ${ }^{1, *}$, N.A. Mansour ${ }^{1}$, Talaat Salah $^{2}$ and Saher Gamal ${ }^{1}$ \\ ${ }^{l}$ Department of Physics, Faculty of Science, Zagazig University, Zagazig, Egypt \\ ${ }^{2}$ Radiation Protection \& Civil Defense Dept., Nuclear Research Center, Egyptian Atomic Energy Authority (EAEA), \\ Cairo, Egypt
}

Received 31 ${ }^{\text {st }}$ March 2019 Measuring radiation dose in a mixed radiation field cannot be performed by using only one dosimeter, Accepted $12^{\text {th }}$ Dec. 2019 but a pair of dosimeters, TLD600 and TLD700 can be successfully used to estimate that radiation dose. Therefore, in the present study, the response of TLD600 and TLD700 to neutron/gamma mixed radiation field was deeply discussed. Moreover, the sensitivity and stability of TLD600 and TLD700 were studied. A strong correlation between TL output signal intensity of TLD700 to gamma dose appeared, with a linear coefficient of $R^{2}=0.99$. Likewise, a strong correlation between TL output signal intensity of TLD600 to gamma dose and neutron irradiation was also evident, with a linear coefficient of $\mathbf{R}^{2}=0.99$. Thus, TLD700 can be applied to measure gamma dose with good sensitivity, while TLD600 can be applied to measure both gamma and neutron dose. Hence, a combination of TLD700 and TLD600 can be successfully employed to assess the radiation doses characterizing a mixed radiation field of neutron and gamma rays.

Keywords: Neutron, Gamma ray, Mixed radiation field, Radiation dose, TLD

\section{Introduction}

Human beings are continuously exposed to ionizing radiation emitted from natural radionuclides existing in the earth's crust, cosmic rays, natural radioactive isotopes, medical diagnostics. Nuclear reactors and radioactive sources used in industry are mostly mixed radiation fields due to the complexity of the charged particle energy spectrum which leads to the deposition of energy [1]. The mixed field can be specified as a compound of two or more different types of radiation or one type of radiation but with different energies; for example, photons and electrons, photons and neutrons or even neutrons with sufficiently different energy share some of the characteristics of a mixed field [2]. In mixed field of the common type of radiation fields, the doses caused by one component could be more significant than the other that can be neglected.
The radiation field around the neutron sources is always a mixed

domain, i.e. various spectra of neutrons (thermal, median, fast and high energy) and gamma rays from neutron capture reaction $(n, \gamma)$ are present $[3,4]$. The radiation dose of gamma/neutron mixed fields for radiation protection purposes has become a necessity in a number of installations since high energy particle accelerators are heavily employed in the medical, industrial and research fields.

To evaluate the radiation dose of a mixed radiation field, the International Commission on Radiation \& Units (ICRU) recommends using a pair of detectors with different responses for each beam component [5]. A pair of thermoluminescence dosimeter (TLD) systems was applied to measure different components in a mixed neutron and gamma irradiation field [6-8]. The essential features of the thermoluminescence are discussed as follows; when ionizing radiation interacts with

Corresponding author: nmmh.6791@gmail.com

DOI: 10.21608/ajnsa.2019.11356.1198

(C) Scientific Information, Documentation and Publishing Office (SIDPO)-EAEA 
the TLD, it deposits all or part of the energy in the material. Some of the atoms in the TLD can produce free electrons from the valence band and holes which are areas that lack electrons after absorbing enough energy [1], as shown in Fig. (1). The impurities in the crystal lattice can trap the free electrons and lock them in the crystal. The crystal structure releases the trapped electrons after being heated to a certain temperature. The released electrons return to the valance band releasing the energy received from the ionization as photons. Holes can also produce photons in an analogous process. The photons can be counted by the use of a photomultiplier tube. The total number of the photons detected is proportional to the number of trapped electrons and holes and, hence, is proportional to ionizing radiation [9]

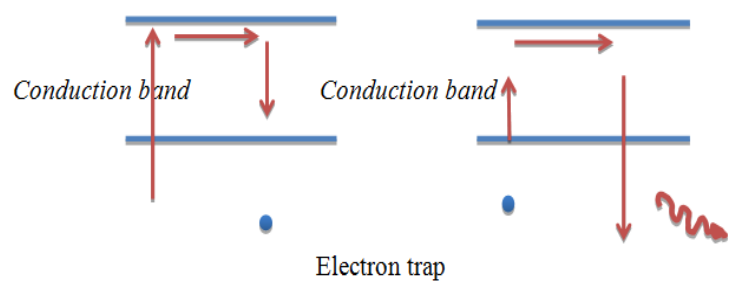

TL photon


a) Irradiation

b) Heating

Fig. (1): Energy level diagram illustrating the principle of TLD

Therefore, the use of a pair of LiF:( $\mathrm{Mg}$, Ti), TLDs of TLD 600 and TLD 700 is an alternative, taking into consideration that they meet these recommendations due to different composition of Lithium isotopes in their composition. The TLD 600, and TLD 700 materials are all LiF:( Mg, Ti) with different lithium isotopes abundance. TLD700 is enriched with ${ }^{7} \mathrm{Li}$ and contains a very small amount of ${ }^{6} \mathrm{Li}$, while the TLD600 is enriched with ${ }^{6} \mathrm{Li}$ to the extent of about 96 percent. The isotope ${ }^{6} \mathrm{Li}$ exhibits a large thermal neutron absorption cross-section, which gives TLD600 the ability to measure thermal neutron $[4,10]$. The effective $\mathrm{Z}$ is 8.2 for both TLD-600 and TLD-700.
The difference between TLD-600 and TLD-700 is the proportion of ${ }^{6} \mathrm{Li}$ and ${ }^{7} \mathrm{Li}$ in the $\mathrm{LiF}$ material. While TLD-600 is made up of $95.62 \%$ of ${ }^{6} \mathrm{LiF}$ which has a large cross-section for neutron and $4.38 \%$ of ${ }^{7} \mathrm{LiF}$, TLD-700 is made up of $0.007 \%$ of ${ }^{6} \mathrm{LiF}$, and $99.99 \%$ of ${ }^{7} \mathrm{LiF}$ [2]. However, all contain different amounts of the ${ }^{6} \mathrm{Li}$ isotope. If a group of dosimeters (all of the same mass and geometry, containing TLD600, and TLD700) is irradiated uniformly with thermal neutrons, there will be clear and easily interpreted differences among the responses of the dosimeter types, with the TLD600 yielding a light output from the thermal neutron dose, while TLD700 would yield virtually no response to the neutrons. TLD600 dosimeters are much more sensitive to thermal neutrons than TLD700 dosimeters enriched in ${ }^{7} \mathrm{Li}$ which interact very weakly with thermal neutrons $[3,4]$.

In the present work, a pair of TLD600 and TLD700 was used for measuring the components of the mixed field of gamma and neutrons. The response of both dosimeters to gamma and neutrons was discussed and their sensitivity as well.

\section{Materials and methods \\ TLD card preparation}

The activated lithium fluoride (LiF) with magnesium (Mg) and titanium (Ti) (LiF: $\mathrm{Mg}, \mathrm{Ti})$, of TLD 600 and TLD 700 in chip form produced by Bicron-Harshaw, USA, was used in the present study to detect neutron and gamma rays. The TLD disc contains two chips of TLD700 and TLD600 mounted on Teflon sheet with aluminum substrates as shown in Fig. (2). Before each irradiation operation and after each use, all dosimeters (TLDs) were annealed (following the producer recommendations) to release electrons that may still be trapped in electron traps and to erase TLD dose memory, to be prepared for subsequent irradiation. The TLDs were placed in an aluminum tray used for the annealing process. According to the manufacturer recommendation, the standard annealing procedure of $\mathrm{LiF}: \mathrm{Mg}$, $\mathrm{Ti}$ material (TLD-600, TLD-700) is a two-temperature process [11]. The TLD600 and TLD700 have been heated at $400{ }^{\circ} \mathrm{C}$ for $1 \mathrm{~h}$, then gradually cooled to room temperature. Afterwards, they are heated again to $100{ }^{\circ} \mathrm{C}$ for $2 \mathrm{~h}$. After that, the TL readout signals of prepared TLD600 and TLD700 were measured with A thermoluminescence reader of type Harshaw 6600 plus. The minimum detectable 
sensitivity of TLD700 and TLD600 were found to be $0.78 \mathrm{nC} / \mathrm{Gy}$ and $0.49 \mathrm{nC} / \mathrm{Gy}$, respectively, i.e. an effective dose of $0.013 \mathrm{mGy}$.

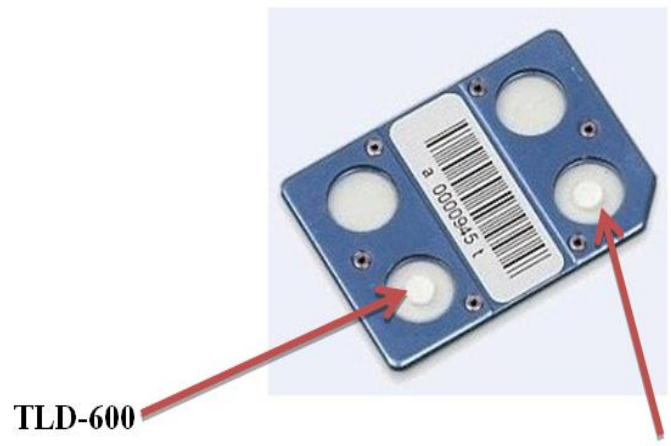

TLD-700

Fig. (2): TLD card

A thermoluminescence reader on type Harshaw 6600 plus readout system was utilized for TL signal measurements, as depicted in Fig. (3). Different TLD cards under investigation, were thermally annealed by Harshaw6600 TL-reader. The annealed TL-cards were ordered into different field sets, each consisting of 10 TLD cards. The TLD cards were treated for $5 \mathrm{~s}$ at a preheat temperature of $150{ }^{\circ} \mathrm{C}$, at a heating rate of $15^{\circ} \mathrm{C} \mathrm{s}$ ${ }^{1}$, up to a maximum temperature of $300^{\circ} \mathrm{C}$ within a reading time of $13.3 \mathrm{~s}$. The glow curve of TLD700 dosimeter irradiated with gamma rays is shown in Fig. (4) and it indicates the intensity of the TL signal of the main acquisition period during the TL-readout of the glow peak. Actually, the intensity of TL readout signal is proportional to the irradiated dose, i.e. the higher irradiated dose, the stronger or largest TL readout signal, as shown in Fig. (4).

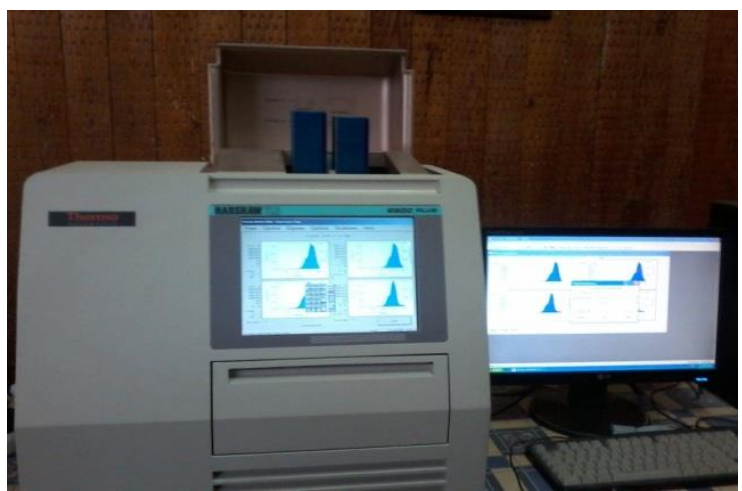

Fig. (3): Harshaw 6600 -plus-TL-reader



Fig. (4): Glow curve of TLD-700 irradiated with gamma ray

Experimental technique

The response of TLD to gamma rays

Different groups of TLD-badges of TLD700 were irradiated with gamma rays at the energy of 661 $\mathrm{keV}$ corresponding to a Cs-137 standard point source, having and activity of $1.5 \times 10^{8} \mathrm{~Bq}$, and with a half-life of 30.2 years. One gram of Cesium137 has an activity of $3.22 \mathrm{TBq}$. The radiation process was carried out in two steps. First, TLD700 chips were exposed to the radiation source for different time intervals ranging from 1 to 6 hours, in a one hour step. Secondly, the TLD700 chips were irradiated at different doses of gamma rays ranging from 10 to 200 rem. In both cases, 24 hours after finishing TLD irradiation (24 hours is required to stabilize prior to reading), TLD was read with Harshaw 6600 plus readout system. In parallel, the gamma dose was also measured with an electronic device, as shown in Fig. (5). A microcomputer-based GM detector model $\mathrm{Hp}-270$, size $3.5 \mathrm{~cm}$ diameter $\times 15.2 \mathrm{~cm}$ length, type ESP-Eberline smart was used for measuring the gamma dose. The energy response of the detector shows a flat response from $40 \mathrm{keV}$ up to $2000 \mathrm{keV}$.

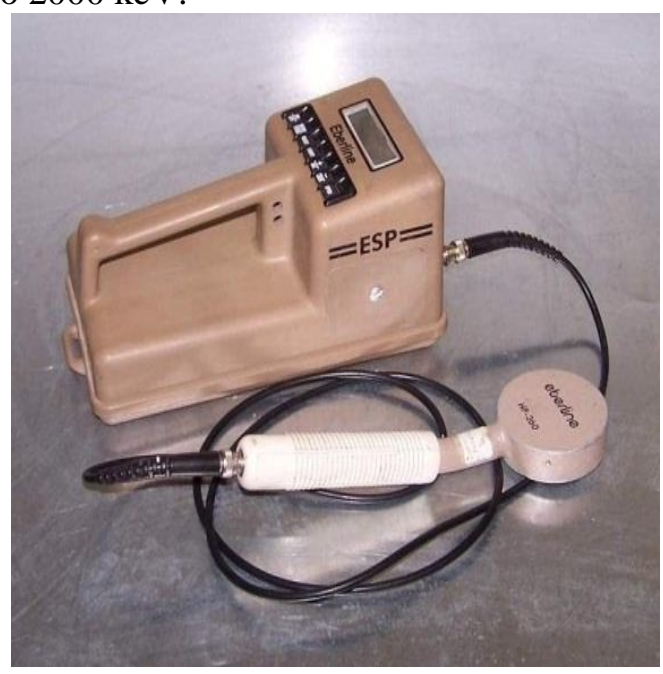

Fig. (5): (a) ESP-1 gamma survey meter 
The response of the Harshaw dosimeter (composed of TLD600 and TLD700) to gamma rays was studied by irradiating both dosimeters to the same gamma dose of $5 \mathrm{mGy}$. Five groups of TLD600 and TLD700 were irradiated to $5 \mathrm{mGy}$, at the same time. Then, after 24 hours all TLD chips were measured with Harshaw TLD-6600-plus-readout system for 5 weeks (three days of each week) to check the electronic stability of reading TLD chips, uniformity, and the response of TLD600 and TLD700.

\section{The response of TLD dosimeter to neutrons}

A number of 40 TLD chips of TLD600 and TLD700 were irradiated at a low dose of gamma rays $(100 \mathrm{mrem})$. After that, the same TLD chips were irradiated with a various fluence of thermal neutrons emitted from Am-Be neutron source with an activity of $5 \mathrm{mCi}$, and average energy of 4.4 $\mathrm{MeV}$. After 24 hours of finishing TLD irradiation (24 hours is required to stabilize prior to reading), TLD was read with Harshaw 6600 plus readout system. At the same time of irradiation process, the neutron dose was measured with a portable neutron survey meter ESP-1. The portable survey meter ESP-1 is a microcomputer base portable radiation survey instrument designed to maneuver with a most radiation detector. ESP-1 is main connected with boron trifluoride $\left(\mathrm{BF}_{3}\right)$ proportional counter detector. The sensor is mainly capsulated inside 8inch diameter polyethylene sphere. Figure (6) presents $\mathrm{BF}_{3}$ neutron detector construction, encapsulated inside the polystyrene Bonner sphere.

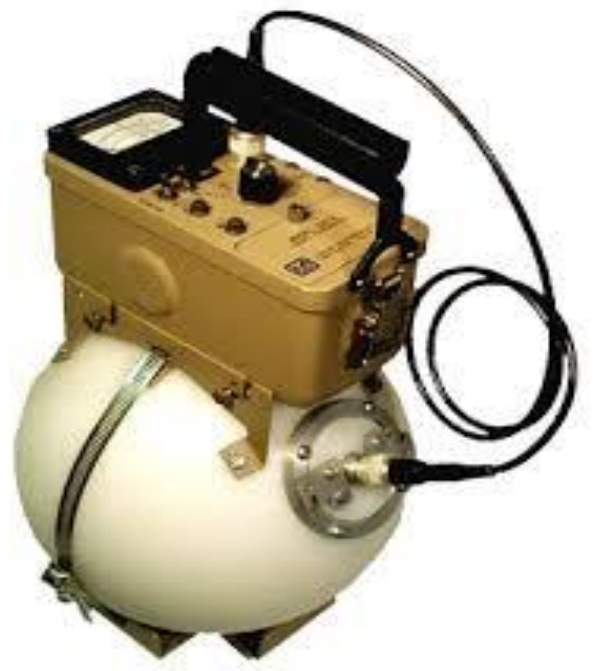

Figure (6): ESP-1 neutron detector

\section{Results and discussion}

The response of TLD700 dosimeter to gamma rays is shown in Fig. (7). It can be noticed that the increase of TL output signal in nano-Coulomb $(\mathrm{nC})$ with the increase of irradiation time or the exposure to the gamma-ray standard source. A strong correlation between the output signal and the irradiation time appeared which implies a strong response of TLD700 dosimeter to gamma dose. A linear function between the output signal and irradiation time was clear, according to a line equation $\mathrm{y}=10.19 \mathrm{x}$ and a linear correlation coefficient of $\mathrm{R}^{2}=0.99$. Likewise, a strong correlation between the output signals of TLD700 and the gamma dose was demonstrated in Fig. (8) which gives a linear growth of output signals with a gamma dose respecting a line equation $\mathrm{y}=$ $2.21+0.22 \mathrm{x}$ and a linear correlation coefficient of $R^{2}=0.99$. This outcome was in agreement with the reported data in the literature related of the response of TLD700 to gamma rays [2].

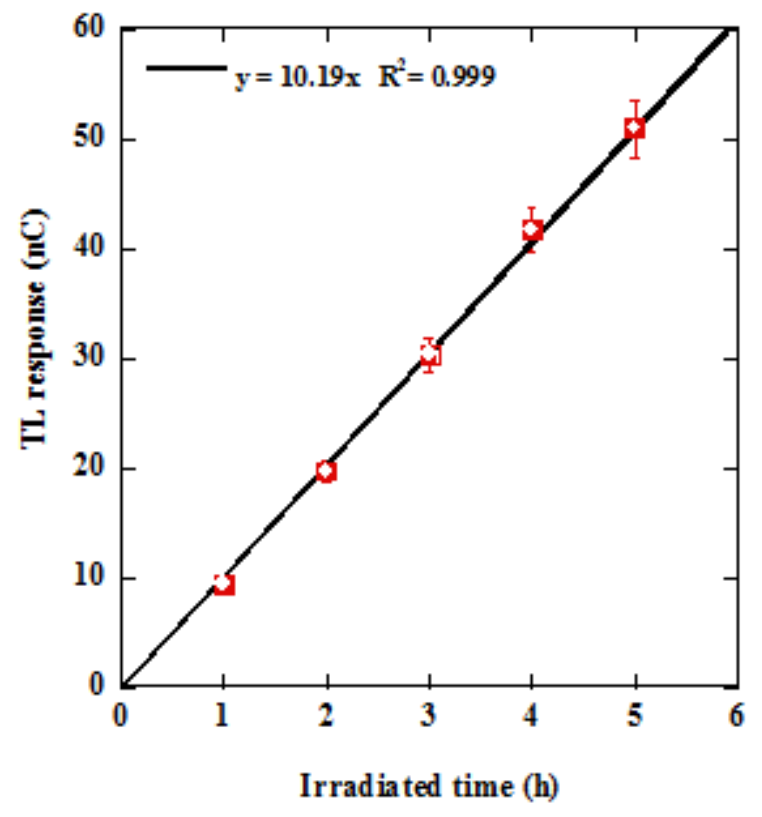

Fig. (7): The relation between the TL-output signal and the irradiated time

After that, five groups of TLD700 and TLD600 were irradiated with $5 \mathrm{mGy}$, their TL output signals were read with Harshaw TLD-6600-plusreadout system for 5 weeks, in a week by week stepwise, three days per week. The output signal response in (nC) is presented in Table (1). By overview, seeing the TL, output signal values, it could be concluded that the TLD700 and TLD600 have the same response to the gamma dose, 
without significant differences. This result is in agreement with the data reported by Cavalieri et al [12]. The mean values of the whole readout signal per week were drawn as shown in Fig. (9). All these results indicated that the used TLD dosimeters have good sensitivity, stability and same response function between TLD700 and TLD600 i.e. any of TLD600 or TLD700 can be used for gamma ray detection, because their response is similar, the difference being insignificant.

The output signals of irradiated TLD600 and TLD700, with a low dose of gamma rays and a various fluence of thermal neutrons, are given in Table (2). The results shown in Table (2), indicated that TLD700 is responded only for gamma rays and it was insensitive to neutron fluence, while TLD600 has good sensitivity for both gamma ray (as discussed before) and thermal neutron fluence. Thus, by subtracting of TL output signal intensity $\left(\mathrm{I}_{\gamma}\right)$ of TLD700 from the corresponding value of TLD600 (the intensity of total response of gamma and neutrons, $\left.\mathrm{I}_{\text {total }}\right)$,

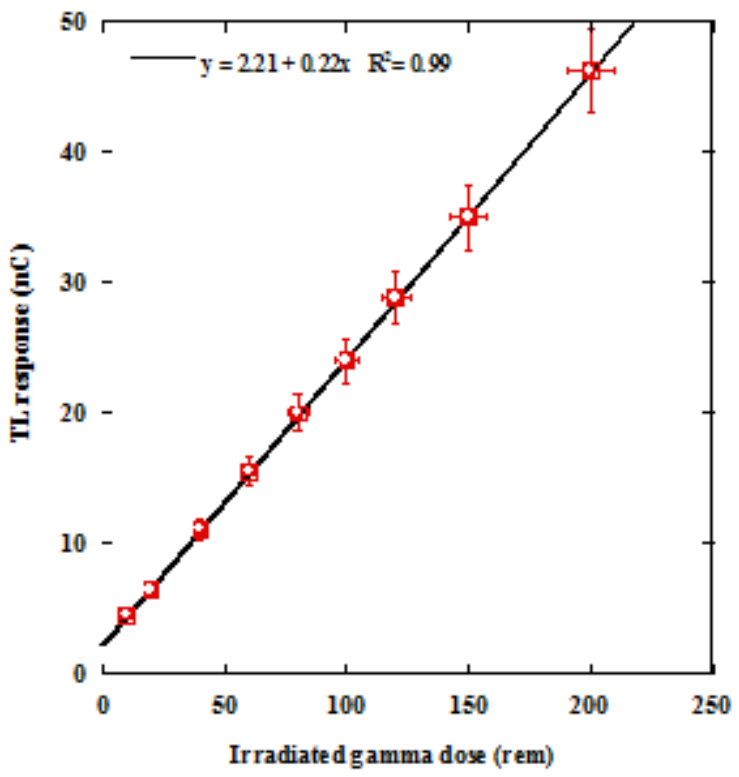

Fig. (8): the relation between the TL-output signal and absorbed gamma dose

Table (1): TL output signal response (nC) of irradiated TLD700 and TLD600 chips

\begin{tabular}{|c|c|c|c|c|c|c|c|c|c|c|}
\hline \multirow{2}{*}{$\begin{array}{c}\mathbf{G} \\
\text { TLD }\end{array}$} & \multicolumn{2}{|c|}{$\begin{array}{c}\text { G-1 } \\
\text { DLD }\end{array}$} & \multicolumn{2}{|c|}{$\begin{array}{c}\text { G-2 } \\
\text { TLD }\end{array}$} & \multicolumn{2}{|c|}{$\begin{array}{c}\text { G-3 } \\
\text { TLD }\end{array}$} & \multicolumn{2}{|c|}{$\begin{array}{c}\text { G-4 } \\
\text { TLD }\end{array}$} & \multicolumn{2}{|c|}{$\begin{array}{c}\text { G-5 } \\
\text { TLD }\end{array}$} \\
\hline & 600 & 700 & 600 & 700 & 600 & 700 & 600 & 700 & 600 & 700 \\
\hline \multicolumn{11}{|c|}{$\overline{1^{\text {st }} \text { week }}$} \\
\hline & 342 & 341 & 342 & 342 & 340 & 342 & 342 & 343 & 341 & 342 \\
\hline & 342 & 343 & 343 & 342 & 341 & 343 & 341 & 344 & 341 & 343 \\
\hline & 343 & 341 & 340 & 340 & 342 & 343 & 342 & 342 & 342 & 341 \\
\hline \multicolumn{11}{|c|}{$2^{\text {nd }}$ week } \\
\hline & 343 & 340 & 340 & 342 & 341 & 342 & 341 & 341 & 340 & 341 \\
\hline & 341 & 341 & 340 & 340 & 340 & 340 & 342 & 340 & 341 & 341 \\
\hline & 341 & 341 & 340 & 343 & 341 & 343 & 340 & 340 & 340 & 341 \\
\hline \multicolumn{11}{|c|}{$3^{\text {rd }}$ week } \\
\hline & 354 & 356 & 356 & 355 & 355 & 354 & 354 & 354 & 350 & 354 \\
\hline & 355 & 356 & 354 & 354 & 353 & 353 & 353 & 353 & 355 & 355 \\
\hline & 355 & 352 & 354 & 353 & 354 & 352 & 351 & 353 & 353 & 352 \\
\hline \multicolumn{11}{|c|}{$4^{\text {th }}$ week } \\
\hline & 348 & 340 & 349 & 349 & 341 & 340 & 342 & 350 & 350 & 349 \\
\hline & 340 & 348 & 348 & 340 & 349 & 348 & 349 & 350 & 350 & 340 \\
\hline & 347 & 347 & 348 & 340 & 349 & 349 & 348 & 350 & 349 & 348 \\
\hline \multicolumn{11}{|c|}{$\overline{5^{\text {th }} \text { week }}$} \\
\hline & 346 & 347 & 346 & 348 & 347 & 338 & 346 & 347 & 346 & 348 \\
\hline & 347 & 347 & 347 & 357 & 346 & 344 & 347 & 347 & 346 & 349 \\
\hline & 349 & 349 & 340 & 346 & 345 & 348 & 346 & 345 & 340 & 346 \\
\hline
\end{tabular}


a neutron output signal response intensity can be obtained $\left(\mathrm{I}_{\mathrm{n}}=\mathrm{I}_{\text {total }}-\mathrm{I}_{\gamma}\right)$, as shown in Table (2) and figure (10). This result implies that TLD600 can be used to measure neutron dose and gamma-ray as well, while TLD700 can be used only for gamma dose. This observation is based on the fact that the high sensitivity of TLD600 to a neutron is due to the high capture cross-section of the ${ }^{6} \mathrm{Li}$ nuclei to thermal neutrons (about 1000 barns) [3]. Hence, a combination of TLD700 and TLD600 can be used to measure a mixed radiation dose of gamma rays and neutron flux.

\section{Conclusion}

The response of the thermoluminescent dosimeters was thouroughly discussed in this study. Likewise, before applied the thermoluminescent dosimeter in the mixed radiation field of neutron and gamma rays, it was calibrated and the sensitivity of TLD at different doses of neutrons and gamma rays was studied. All the results indicated that the used TLD dosimeters have a good sensitivity, stability and same response function between TLD700 and TLD600 to gamma ray i.e. any of TLD600 or TLD700 can be used for gamma ray detection, because their response is similar, the difference being insignificant. TLD700 has a good response to gamma rays only, while TLD600 has a good response to both gamma-ray and neutron dose. Hence, a combination of TLD700 and TLD600 can be employed to assess the radiation doses of a mixed radiation field consisting of neutron and gamma rays.

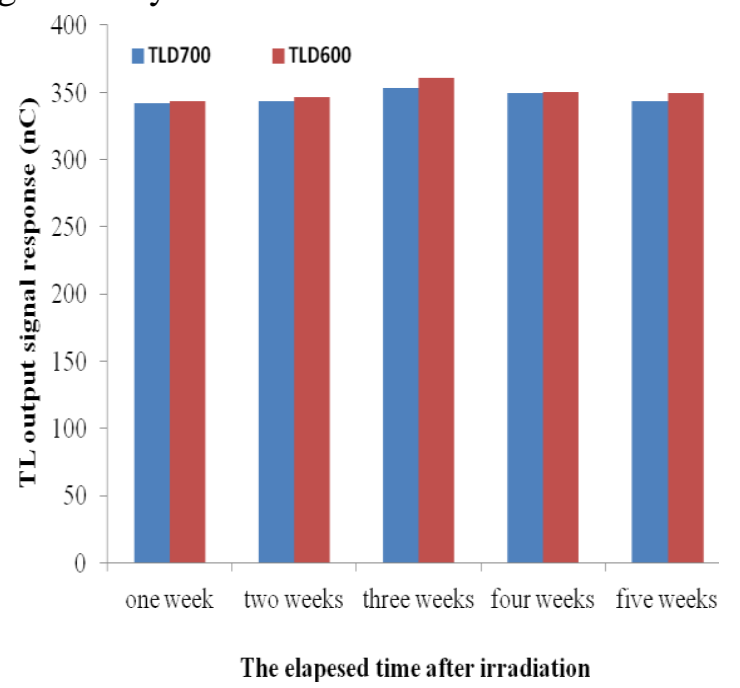

Fig. (9) The average values of the whole readout signal per week for five weeks

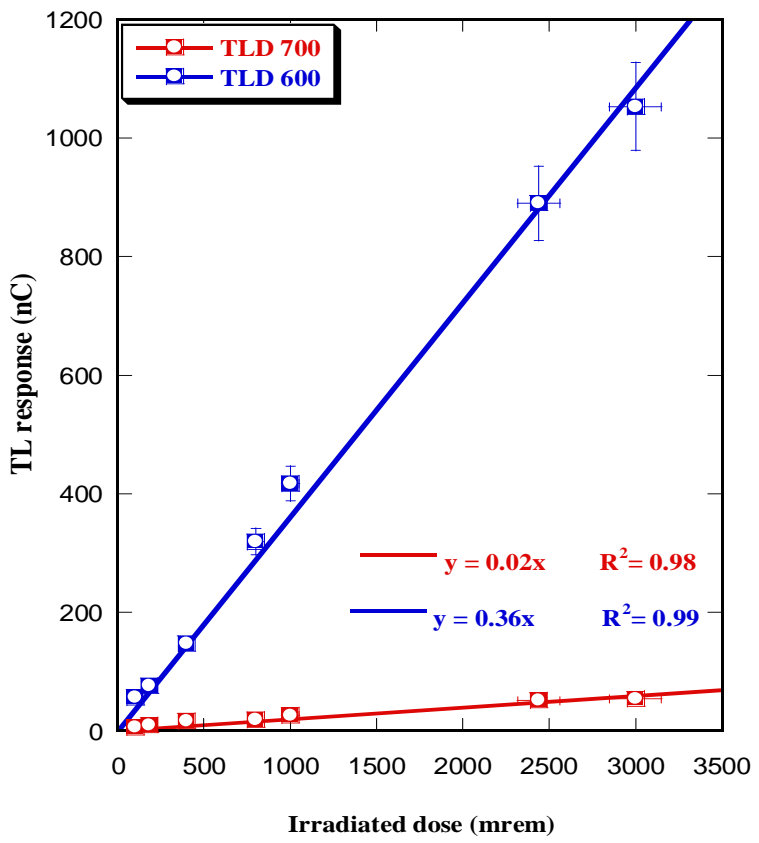

Fig. (10) TLD response to thermal neutron and gamma ray

Table (2): The differential of output intensity response of TLD700 and TLD 600 to gamma/neutron mixed field (nC)

\begin{tabular}{|c|c|c|c|}
\hline $\begin{array}{l}\text { Dose } \\
\text { (mrem) }\end{array}$ & $\begin{array}{l}\text { Intensity } \\
\left(\mathrm{I}_{\gamma}\right) \text { of } \\
\text { TLD-700 } \\
(\mathbf{n C})\end{array}$ & $\begin{array}{l}\text { Intensity } \\
\left(\mathrm{I}_{\text {total }}\right) \text { of } \\
\text { TLD-600 } \\
\text { (nC) }\end{array}$ & $\begin{array}{l}\text { Intensity } \\
\left(\mathrm{I}_{\mathrm{n}}\right) \\
\text { Thermal } \\
\text { neutron }\end{array}$ \\
\hline 100 & $6.8 \pm 1.6$ & $56.6 \pm 9.3$ & $49.7 \pm 7.7$ \\
\hline 183 & $10.4 \pm 0.7$ & $75.8 \pm 8.4$ & $65.43 \pm 7.7$ \\
\hline 400 & $16.1 \pm 4.0$ & $147.9 \pm 14.1$ & $\begin{array}{l}131.8 \\
10.1\end{array}$ \\
\hline 800 & $19.1 \pm 2.2$ & $319.4 \pm 51.8$ & $\begin{array}{l}300.4 \\
49.6\end{array}$ \\
\hline 1000 & $26.6 \pm 3.5$ & $417.2 \pm 57.7$ & $\begin{array}{l}390.5 \\
54.2\end{array}$ \\
\hline 2440 & $51.2 \pm 16.2$ & $900.0 \pm 68.4$ & $\begin{array}{l}838.5 \\
52.2\end{array}$ \\
\hline 3000 & $54.1 \pm 13.3$ & $1053 \pm 63.6$ & $999 \pm 50.3$ \\
\hline
\end{tabular}

\section{Reference}

1- Saad N., (2012) The Application of Experimental Microdosimetry Mixed Field Neutron/Gamma Dosimetry. Thesis for the Degree of Master of Applied Science In Nuclear Engineering. University of Ontario Institute of Technology (Canada), Publication Number: AAT MR92030; ISBN: 9780494920305; Source: Masters Abstracts International, Volume: 51-04.; 117 p. 2- Yanan C., (2013) Characterization of TLD-600 and TLD-700 and acceptance testing of new X-RAD 160 biological X-Ray irradiator. The thesis of the degree of master in medical physics, Duke University. 
3- Triolo A., Marrale M., Brai M., (2007) Neutrongamma mixed field measurements by means of MCPTLD600 do,imter pair. Nuclear Instruments and Methods in Physics Research B 264 ,183-188.

4- Rabie N., Hassan G.M., El-Sersy A.R. and Ezzat M., (2010) Study of the improvement of TLD cards for personal neutron dosimetry. Radiation Effects \& Defects in Solids: Incorporating Plasma Science \& Plasma Technology Vol. 165, No, 329-336.

5- International Commission on Radiation Units and Measurements, (1984) Neutron dosimetry for biology and medicine.

6- Sayed A.M., (1975) Investigation of physical parameters affecting the used of different types of dosimeters in mixed field personnel dosimetry. The thesis of the degree of philosophy doctor in physics, ElAzhar University, Cairo.

7- Durrani S.A. and Khan H.A.,(1975) Solid state track detectors for neutron image recording. Radiography with Neutrons" (Ed. Hawkesworth M. R.), pp. 71-77. BNES, London.

8- Kamenopoulou V., Carinou E. and Stamatelatos I. E., (2001) Personal neutron dosimetry at a research reactor facility. Radiation Protection Dosimetry, Vol. 96, pp. 197-200.

9- Knoll, Glenn F., (1999) Radiation detection and measurement (3rd ed.). New York: Wiley.

10- Haiyong J., Kun Jai Lee, Jang-Lyul Kim, (2003) A personal thermoluminescence dosimeter using LiF: Mg, $\mathrm{Cu}, \mathrm{Na}, \mathrm{Si}$ detectors for photon field. Applied Radiation and Isotopes 59, pp. 87-93.

11- Thermo Scientific Product Overview Materials and Assemblies for Thermoluminescence Dosimetry.

12- Cavalieri T.A., Castro V.A., and Siqueira P.T.D., (2013) Differences in TLD600 and TLD700 glow curves derived from district mixed gamma/ neutron field irradiations. International Nuclear Atlantic Conference. Recife, PE, Brazil, November 24-29. 